\title{
The Management of Innovation Projects' Implementation in Production-and-Economic Systems: The Use of Prognostic Models for the Risk Metrics of Obtained Decisions
}

\author{
Leonid Mylnikov* \\ Perm National Research Polytechnic University, 29 Komsomolsky Prospekt, 614990 Perm, Russia \\ ${ }^{*}$ Corresponding author
}

\begin{abstract}
The article describes a criteria-based approach to calculate the total risk obtained by the use of forecasts in prognostic models in order to solve tasks in production-andeconomic systems; this chapter also outlines the difficulty of recording risks received by the use of prognostic functions and values in mathematical operations and by applying the set of prognostic functions and values with various risk values.
\end{abstract}

Keywords-component; manufacturing planning, risk evaluation, simulation modeling, project management, production-andeconomic system

\section{INTRODUCTION}

Project implementation methods already exist (for example [1]). However, the implementation of innovation projects for goods in production-and-economic systems (PES) involves operational management over a group of projects within already established PES processes to measure efficiency [2] (i.e. is based on traditional hierarchy of indicators (DuPont model for instance) and has one target indicator - profit, which is not sufficient for implementing innovation projects for goods since the management of such projects requires a whole set of sometimes contradicting indexes);introduction and control over new projects [3] (helps consider the multidimensionality of innovation projects, yet is not aimed at the development and growth of PES profitability, neither takes into account the optimal nature of obtained decisions); collection and analysis of input data, data about production processes, output data and production outcome [4] (as a rule, collecting general system data is not sufficient to determine the data that will be considered enough to describe a certain project); information infrastructure [5].

Taking into consideration the above-mentioned, parameters and indicators can be defined based on project types.

Currently, we can talk about a new generation of planning and management tasks solved by the use of prognostic data. Such tasks comprise the tasks for volume scheduling in production planning of goods, procurement planning of components and parts, expected demand-based pricing etc. (depending on the applied methods and the phase of project or system implementation as well as parameters and indexes used to take managerial decisions) [6]). Such tasks particularly require the use of criteria-based methods and prognostic data with criteria functions and time parameter restrictions. Their structure is demonstrated in the Fig. 1.

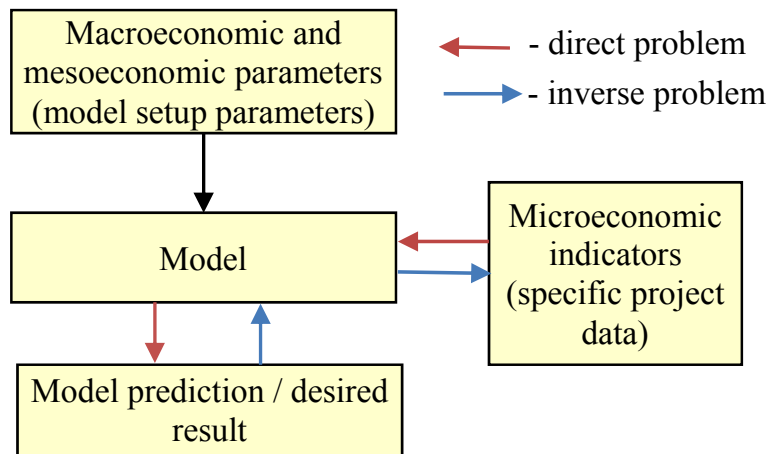

FIGURE I. PLACE AND ROLE OF VARIOUS TYPES OF PARAMETERS THAT CHARACTERIZE INNOVATION PROJECTS.

For the mathematical formalization we need to define calculation parameters $Z=A X$, where $Z$ - parameter vector (including computable parameters), $A$ - identity matrix, $X$ - the vector of model parameters (including the parameters obtained by prognostic data with the risk $R(\varepsilon)$ and with the accuracy $\varepsilon$ ).

$$
\begin{gathered}
J\{Z\} \rightarrow \text { opt } \\
f_{i}(Z): G_{i}, i=\overline{1, n},
\end{gathered}
$$

where : - is one of operations $>,<, \geq, \leq,=, \neq ; i$ - the number of used restrictions, forecast-based model parameters; $G_{i}$ - values for restrictions; $f_{i}(Z)$ - the function with parameters. Look for the examples of certain formalizations in the references (for instance, [6] and [7]).

The task is solved by a function. However, obtained solution will show different risk metrics depending on the profoundness of planning; furthermore, the solutions and risk metrics will change every single time as new data come.

In periodicals we can find approaches to measure risks for a solution itself or a prognostic function [8].

Total risks obtained for each single model will be different as they depend on the gained mathematical formalization. 


\section{FORECAST-BASEd Risk METRICS OF PARAMETER VALUES}

Forecasting can be carried out only with a certain degree of accuracy; herewith, the longer time sequence we take for forecasting, the lower is the accuracy of prognostic data (the intervals of potential deviations will increase). For the parameters that obey normal distribution law the margin of deviations can be calculated by taking into account the maximum degree of an error (fault) when the forecast can be deemed reliable:

$$
\mathrm{F}=\sum_{j=1}^{n} E_{i}^{2}
$$

where $E_{i}=Y_{i}^{T}-Y_{i}^{*} \$$ - the margin between forecasting and real values, $Y_{i}^{T}-$ prognostic data, $Y_{i}^{*}$ - data about the change of parameter size, obtained during project implementation (experimental data used for forecasting).

Let us determine $\sigma=\frac{F}{n}$, where $n$ - the number of experimental points.

Then, according to normal distribution law the adequacy of hypothesis is supported by reaching the interval $Y^{*}-\sigma \leq Y^{T} \leq$ $Y^{*}+\sigma-68 \%$ experimental points and more, the interval $Y^{*}-$ $2 \sigma \leq Y^{T} \leq Y^{*}+2 \sigma$ - not less than $95 \%$ experimental points, the interval $Y^{*}-3 \sigma \leq Y^{T} \leq Y^{*}+3 \sigma \quad-$ not less than $99 \%$ experimental points.

Due to a vague nature of a described forecast let us set forecasting values as fuzzy figures. Each value of a forecasting curve is assigned a corresponding membership function. Thereby, we mathematically describe the market share connected with the use of forecasts. For the determination of this market share we need to define the size of risk.

To clarify, let us assume that a risk is calculated by a certain number of factors affecting the risk:

$$
r=1-\frac{a}{a^{*}}
$$

where $a$ - the fixed, planned value of unit cost without risk ; $a^{*}$ the defined index of unit cost.

The defined index used in this formula is to be determined by either expert evaluations or forecast margins obtained by various methods. These data can be received with help of diverse original information. These data are used to define the range of margins for a crucial parameter (i.e. the risk calculated by this method will unequivocally be placed in this range that generates a fuzzy set); membership function is built upon Gaussian function (used to describe normal distribution law).

To specify function parameters, let us use Gaussian function $\left(\mu=\frac{1}{\sigma \sqrt{2 \pi}} e^{-\frac{(x-c)^{2}}{2 \sigma^{2}}}\right)$ and obtained margins. $c$ can be assigned, if known, retrospective data or most accurate forecasting data as well as arithmetic average of gained forecasts (same like W.S. Gosset (Student) treated measurement results). Let us use the property of full width at half amplitude to find the margin $\sigma$ :

$$
\sigma=\frac{c_{\max }}{\sqrt{2 \ln 2}}
$$

where $c_{\max }=\lim _{1 \leq j \leq n}\left|c-c_{i}\right|$, where, respectively, $i$ - the number of used alternative margins for $c$ obtained with help of forecasts and expert evaluations. Membership function looks like then as follows (for the parameters that obey normal distribution law):

$$
\mu(x)=\frac{\sqrt{\ln 2}}{c_{\max } \sqrt{\pi}} e^{-\frac{\ln 2(x-c)^{2}}{c_{\max }^{2}}}
$$

For the parameters that do not obey normal distribution law we can apply triangular functions, generic Bell function, sigmoid function for asymmetric distributions and so on.

Based on the membership function that already encompasses potential risky events affecting each of the margins, the margin of risk metrics is calculated [8] for the examined parameter value:

$$
r=1-\frac{\int_{\alpha}^{\beta} \mu_{1}(x) d x}{\int_{\alpha}^{\beta} \mu_{2}(x) d x}
$$

where $r$ - the risk metrics; $\mu_{1}(x)$ and $\mu_{2}(x)-$ membership functions for various margins of $c$ (for instance, $c_{1}$ - the margin of retrospective data, $c_{1}$ - the arithmetic average of obtained margins); $\alpha, \beta$ - boundaries of margins' range).

The calculation of the margin of risk metrics for obtained forecast values generates the function of risk metrics in a tabular form $r(c)$.

The described risk metrics designed for a parameter encompasses the impact of other margins that affect a parameter, since their impact was considered in the forecast.

The obtained risk metrics does not consider the risks of previous phases and neither takes into account risk metrics for a project or a system since they are described by a set of independent parameters.

\section{Risk MEtrics By PhASEd ProJECt IMPLEMENTATION}

Since there is a set of parameters we can define decision points based on the characteristics and phases of their progress (sales volume, price change and other parameters described by innovation curves), statistical data or prognostic data as well as PES data (equipment service intervals, internal technology cycles etc.).

By phased project implementation or by the development of companies we need to measure the risks connected with the attainability of results in their consecutive implementation (including different scenario of potential development). Therefore, risk margin becomes dependent on the risk margin of previous phases:

$r\left(c_{i}^{(0)}, c_{i}^{(1)}, \ldots, c_{i}^{(m)}\right)=r\left(c_{i}^{(0)}\right) r\left(c_{i}^{(1)} \mid c_{i}^{(0)}\right), \ldots, r\left(c_{i}^{(m)} \mid c_{i}^{(m-1)}\right)$.

Risk metrics is the probability function $r(P)$ [9] that indicates the probability of planned margins over a confidence interval. Based on (1) it can be determined as $P=1-r$.

Hence, the risk of attaining the values of the risk function set in a tabular form should be examined as a chain of interrelated events. Given that, the probability of interrelated events $c_{1}$ and $c_{2}$ provided that $c_{2}$ comes after $c_{1} P\left(c_{1} \mid c_{2}\right)=P\left(c_{1}\right)+P\left(c_{2}\right)-$ 
$P\left(c_{1}\right) P\left(c_{2}\right)$, from whence we receive $r\left(c_{1} \mid c_{2}\right)=1-P\left(c_{1}\right)=$ $1-r\left(c_{1}\right)+1-r\left(c_{2}\right)-r\left(c_{2}\right)-r\left(c_{1}\right)+r\left(c_{1}\right) r\left(c_{2}\right)=2-$ $2 r\left(c_{2}\right)-2 r\left(c_{1}\right)+r\left(c_{1}\right) r\left(c_{2}\right)$.

These values can be used in probabilistic models (in particular, Markov chains [10]).

Let us assume that we would like to measure the risk connected with the attainability of a number of consequent targets $s_{1}, s_{2}, \ldots, s_{n}$ that are assigned specific values of indexes (for instance, set by various forecast values), then the probability $p_{i}^{(0)}$ - shows that we are placed in the state $s_{i}$ and that the state totally corresponds to the expected state (defined by the risk values of previous phases (for example, $r\left(c_{i}^{(1)} \mid c_{i}^{(0)}\right)$ ). Let us consider $p_{i j}$ - as the probability of the shift from the state $s_{i}$ into the state $s_{j}$ (defined by the values $\left(c_{i}^{(0)}\right)$ ), and $p_{i}^{(1)}-$ is the probability for achieving the state $s_{i}$. Hence, it comes from [10] as follows:

$$
\begin{aligned}
& \left(p_{1}^{(1)}, p_{2}^{(1)}, \ldots, p_{n}^{(1)}\right)=\left(p_{1}^{(0)}, p_{2}^{(0)}, \ldots, p_{n}^{(0)}\right) \times \\
& \left(\begin{array}{crll}
p_{11} & p_{12} & \cdots & p_{1 n} \\
p_{21} & p_{22} & \cdots & p_{2 n} \\
\vdots & \vdots & \ddots & \vdots \\
p_{n 1} & p_{n 2} & \cdots & p_{n n}
\end{array}\right)
\end{aligned}
$$

and the task is to determine the probabilities of how one state comes

into another state.

By determining risk metrics for a model that is set with help of mathematical formalization when the parameters are interrelated by the described mathematical operations we encounter another task, and namely the task to create a general model to calculate risks.

For simplicity, let us assume that a system model is described by the functions of independent parameters $f\left(c_{1}, c_{2}\right)$. Then, taking into account that $P=1-r$ for independent $c_{1}$ and $c_{2} P\left(c_{1} c_{2}\right)=P\left(c_{1}\right) P\left(c_{2}\right)$. From whence we receive $r\left(c_{1} c_{2}\right)=$ $1-P\left(c_{1} c_{2}\right)=1-P\left(c_{1}\right) P\left(c_{2}\right)=r\left(c_{2}\right)+r\left(c_{1}\right)-r\left(c_{1}\right) r\left(c_{2}\right)$.

Therefore, as a result we receive total risk metrics for prognostic models used to support managerial decisions.

\section{CONCLUSION}

Production-and-economic systems and the description of innovation projects require a vast number of parameters that are described by innovation curves [11]. These curves have progress phases; except that, production-and-economic systems also have internal cycles. Shift points from one phase to another phase together with the points that indicate the end and the start of internal cycles of production-and-economic system generate multiple points for decision making [6].

The formalization of tasks in terms of optimization tasks can be confronted with the problem NP - completeness. Particularly, the task of joint examination of market selection and volume planning in production is subject to these tasks and it will be solved only if we fix separate factors [12] or apply the methods of numerical design. In this case, the margin of risk metrics model can be deemed the single objective index that can be used to consider the quality of made decisions; by simplifying the task when risk metrics and received faults are considered all together, this margin can be used to decide the expediency of the solution in the set circumstances.

Consequently, risk metrics is specific in the way it is applied to calculate the perspectives for project progress and to design models to support managerial decisions within the implementation of innovation projects in production-andeconomic systems.

The conclusions received in this article can be used since there is risk metrics for all the parameters and there is a model designed for all the parameters, that we would like to consider; more than that, there should be a need to carry out a study that requires the selection of parameters sufficient to measure an integral risk of an innovation project.

\section{ACKNOWLEDGMENT}

The author thanks the government of Perm Krai for the support of the project for "Development of software and economic and mathematical models for supporting innovation project management processes in production systems", which is being implemented in accordance with decree №166-ח of 06.04.2011.

The reported study was partially supported by the Government of Perm Krai, research project No. C-26/058.

\section{REFERENCES}

[1] C. Hoffmann, S. Lennerts, C. Schmitz, W. Stölzle, and F. Uebernickel, Eds., Business Innovation: das St. Galler Modell. Wiesbaden: Springer Gabler, 2016.

[2] R. Foster, L. Linden, R. Whiteley, and A. Kantrow, "Improving the Return on R\&D-II," Res. Manag., vol. 28, no. 2, pp. 13-23, 1985.

[3] I. C. Kerssens-van Drongelen and A. Cooke, "Design principles for the development of measurement systems for research and development processes,” R Manag., vol. 27, no. 4, pp. 345-357, Oct. 1997.

[4] M. Brown and R. Svenson, "Measuring R\&D Productivity," Res. Technol. Manag., vol. 41, no. 6, pp. 30-35, 1998.

[5] M. Kütz, "IT-Prozesse mit Kennzahlen steuern," Control. Manag. Rev., vol. 58, no. 7, pp. 86-93, Mar. 2014.

[6] R. A. Faizrakhmanov and L. A. Mylnikov, "The foundations of modeling management processes for innovation projects in production-economics systems," Autom. Doc. Math. Linguist., vol. 50, no. 3, pp. 84-90, May 2016.

[7] L. Mylnikov, "Particularities of Solving the Problems of Support for Managerial Decision Making in Production and Economic Systems Using the Statistical Data," Int. J. Econ. Financ. Issues, vol. S8, no. 6, pp. 1-11, 2016.

[8] L. Mylnikov, "Risk Evaluation in Manufacturing Organization Tasks for Product Technological Projects and Establishment of Project Portfolio for Production Systems," in Proceedings of the 2016 International Conference on Applied mathematics, simulation and modelling, Beijing, China, 2016, pp. 399-402.

[9] F. P. Esposito and M. Cummins, "Multiple Hypothesis Testing of Market Risk Forecasting Models: MHT of Market Risk Forecasting Models," J. Forecast., vol. 35, no. 5, pp. 381-399, Aug. 2016.

[10] J. A. Anderson, Discrete mathematics with combinatorics. Upper Saddle River, N.J: Prentice Hall, 2001.

[11] M. Amberg and Mylnikov, L., "Innovation project lifecycle prolongation method," Innov. Knowl. Manag. Twin Track Econ. Chall. Solut., no. 1-3, pp. 491-495, 2009.

[12] W. Van den Heuvel, O. E. Kundakcioglu, J. Geunes, H. E. Romeijn, T. C. Sharkey, and A. P. M. Wagelmans, "Integrated market selection and production planning: complexity and solution approaches," Math. Program., vol. 134, no. 2, pp. 395-424, Sep. 2012. 